\title{
Staphylococcus saccharolyticus
}

National Cancer Institute

\section{Source}

National Cancer Institute. Staphylococcus saccharolyticus. NCI Thesaurus. Code C86769.

A species of facultatively anaerobic, Gram positive, cocci shaped bacteria in the phylum Firmicutes. This species is positive for catalase and neg ative for oxidase, coagulase, and urease. It can ferment mannose, and fructose, but not sucrose, maltose, xylose, lactose, trehalose, mannitol, xylitol, arabinose, or cellobiose. S. saccharolyticus is part of the normal human skin flora but may be a human pathogen that can cause endocarditis. 